\title{
Recovery of Image through Alamouti Channel with Incorporation of RSA Algorithm
}

\author{
Aninda Majumder, Mohammad Raihan Ruhin, Tahsina Hashem, Md. Imdadul Islam \\ Department of Computer Science and Engineering, Jahangirnagar University, Dhaka, Bangladesh \\ Email: ani_1860@yahoo.com, raihanruhin@gmail.com, tahsina.hashem@juniv.edu, imdad@juniv.edu
}

Received 31 December 2015; accepted 12 February 2016; published 15 February 2016

Copyright (C) 2016 by authors and Scientific Research Publishing Inc.

This work is licensed under the Creative Commons Attribution International License (CC BY). http://creativecommons.org/licenses/by/4.0/

(c) (i) Open Access

\begin{abstract}
In many applications, it is necessary to transmit images at a remote station, where wired Internet service is not available. In this case, wireless local loop (WLL) can help in making wireless link between one end node of the internet and remote service center. In such link, the communication is heavily affected by large and small scale fading; hence the received signal experiences huge distortion in case of forward error correction. Otherwise, huge service delay arises due to frequent negative acknowledgements. To combat the situation, we can choose Alamouti channel of full rate and fully orthogonal space-time block code (OSTBC). Our aim is to transmit images through Alamouti channel and to observe the quality of the recovered image, in context of bit error rate (BER). We have also observed the impact of fading and additive white Gaussian noise (AWGN) on the image without application of error correction or detection technique of channel coding. To ensure security, we apply the RSA algorithm on each pixel prior transmitting and decrypt them at the receiving end, where we found no impairment from the algorithm. Finally, we observe that the relative performance of the system changes digital modulation schemes.
\end{abstract}

\section{Keywords}

Quotient and Remainder Matrix, Alamouti Simulator, Multiple-Input Single-Output (MISO), Image Encryption and Decryption, BER and Discrete Wavelet Transform (DWT)

\section{Introduction}

Two most important limitations of wireless communication are: the range or length of link and data rate (related to channel capacity). To overcome these limitations, researchers have given their effort on space diversity and 
multicarrier parallel transmission called orthogonal frequency division multiplexing (OFDMA) of [1] [2]. A number of algorithms are proposed on transmitting side in [2]-[7] for attaining space diversity hence getting high signal-to-noise ratio (SNR) at receiving end. A communication model, where space-time block code (STBC) at the physical layer, with channel coding (linear block code) at the data link layer is shown in [8]-[10] and performances were measured in context of BER. A diversity coding can be used in multiple-input multipleoutput (MIMO) to provide high data rate without requiring additional bandwidth and transmit power. It is noted that Alamouti space-time block coding has showed good performance than other techniques comparatively. This motivates us to use Alamouti channel for transmitting different types of images like "medical images", "secured watermark", "biometric identification" at a remote station.

Several digital modulation schemes i.e. binary phase-shift keying (BPSK), quadrature phase-shift keying (QPSK), quadrature amplitude modulation (QAM) are used to measure the performance of the system in [11] [12]. It is observed that a well-known cryptographic algorithm RSA [13] is used by researchers [7] for ensuring security of the transmitted message. Research [14]-[16] on MIMO with maximal ratio combining (MRC), selection combining (SC) and equal gain combining (EGC) diversity reception over several correlated and uncorrelated fading channels is also going on.

In this research, Alamouti space-time block coding is applied on transmission channel with incorporation of RSA, for successful recovery of an image. We summarize our key contributions as follows:

- We proposed an algorithm to transmit image through Alamouti channel because of its full spatial diversity gain and observe the quality of recovered image by measuring the bit error rate.

- Furthermore, to ensure security we apply the RSA algorithm on each pixel of the image.

- We perform a number of experiments over Rayleigh fading channels by varying SNR and by applying 16-QAM modulation schemes in order to measure the performance of the system.

The remainder of this paper is organized as follows. Section 2 presents our proposed algorithm of recovering image through Alamouti scheme with incorporation of RSA. In Section 3, we present our experimental results in details with respect to a variety of parameters. Finally, Section 4 concludes the paper with future research directions.

\section{System Model}

The space-time block code (STBC) is used to achieve orthogonality among transmitted symbols of individual antenna of the MIMO system [17] [18]. Alamouti scheme is a full rate OSTBC where two transmits and one receive antenna are used provided the receiver has the knowledge of channel gain. When more than two antennas are used, then orthogonality is possible but transmission rate become $1 / 2$ or $3 / 4$, for example, sporadic code.

\subsection{Full Rate STBC}

Full rate STBC is also possible for the case of transmitted antennas, $n_{T}=2^{k}, k=2,3,4, \cdots ;$ but full orthogonality is not possible and the scheme is called QSTBC explained in [19]-[21]. We can apply zero forcing (ZF) on QSTBC to convert its full orthogonal [21] at the expense of noise. In OSTBC the multiplication channel matrix and its conjugate transpose produces a diagonal matrix i.e. the off-diagonal elements are zero. In case of quasiorthogonal, the result produces a few non-zero off-diagonal elements like below:

$$
\left[\begin{array}{cccc}
h_{1} & h_{2} & h_{3} & h_{4} \\
h_{2}^{*} & -h_{1}^{*} & h_{4}^{*} & -h_{3}^{*} \\
h_{3} & h_{4} & h_{1} & h_{2} \\
h_{4}^{*} & -h_{3}^{*} & h_{2}^{*} & -h_{1}^{*}
\end{array}\right]\left[\begin{array}{cccc}
h_{1} & h_{2} & h_{3} & h_{4} \\
h_{2}^{*} & -h_{1}^{*} & h_{4}^{*} & -h_{3}^{*} \\
h_{3} & h_{4} & h_{1} & h_{2} \\
h_{4}^{*} & -h_{3}^{*} & h_{2}^{*} & -h_{1}^{*}
\end{array}\right]^{H}=\left[\begin{array}{cccc}
\alpha & 0 & \beta & 0 \\
0 & \alpha & 0 & \beta \\
\beta & 0 & \alpha & 0 \\
0 & \beta & 0 & \alpha
\end{array}\right]
$$

where, each element of the matrix $h_{i}$ indicates the channel gain, $\alpha=\left|h_{1}\right|^{2}+\left|h_{2}\right|^{2}+\left|h_{3}\right|^{2}+\left|h_{4}\right|^{2}$ and $\beta=h_{1} h_{3}^{*}+h_{3} h_{1}^{*}+h_{2} h_{4}^{*}+h_{4} h_{2}^{*}$

In zero forcing decoding technique, the quasi-orthogonal reception is made fully orthogonal. Here we can eliminate interferences at the expense of noise. From MIMO scheme, the received signal vector is

$$
\boldsymbol{r}=\left[r_{1} r_{2} r_{3} r_{4}\right]=\boldsymbol{H} \boldsymbol{x}+\boldsymbol{n}
$$


The estimated received signal vector under $\mathrm{ZF}$ is

$$
\begin{aligned}
\hat{\boldsymbol{Y}} & =\left(\boldsymbol{H} \boldsymbol{H}^{H}\right)^{-1} \boldsymbol{H}^{H} \boldsymbol{r}=\left(\boldsymbol{H} \boldsymbol{H}^{H}\right)^{-1} \boldsymbol{H}^{H}(\boldsymbol{H} \boldsymbol{x}+\boldsymbol{n}) \\
& =\left(\boldsymbol{H} \boldsymbol{H}^{H}\right)^{-1}\left({ }^{1} \boldsymbol{H}^{H} \boldsymbol{H}\right) \boldsymbol{x}+\left(\boldsymbol{H} \boldsymbol{H}^{\mathrm{H}}\right)^{-1} \boldsymbol{H}^{\mathrm{H}} \boldsymbol{n} \\
& =\boldsymbol{I}_{4} \boldsymbol{x}+\left(\boldsymbol{H} \boldsymbol{H}^{H}\right)^{-1} \boldsymbol{H}^{H} \boldsymbol{n} \\
& =\boldsymbol{x}+\boldsymbol{n}^{\prime} ; \text { where } \boldsymbol{n}^{\prime}=\left(\boldsymbol{H} \boldsymbol{H}^{\mathrm{H}}\right)^{-1} \boldsymbol{H}^{\mathrm{H}} \boldsymbol{n} \\
& =\left[x_{1} x_{2} x_{3} x_{4}\right]^{\mathrm{T}}+\left[n_{1}^{\prime} n_{2}^{\prime} n_{3}^{\prime} n_{4}^{\prime}\right]^{\mathrm{T}}
\end{aligned}
$$

Now we have to go for better STBC technique, which provides orthogonality among the received symbols at the same time noise is not enhanced like ZF algorithm of above. In this case, we can choose the simplest possible orthogonal STBC of two antenna systems, called Alamouti scheme discussed in next subsection.

\subsection{Alamouti Scheme}

Alamouti scheme is a space-time block code under MISO, where the transmitting end uses two antennas and the receiver side has only one antenna, hence the system has no scope of using combining scheme. It is the only full rate orthogonal STBC discussed in [22]. In Alamouti scheme two symbols $\tilde{S}_{1}$ and $\tilde{S}_{2}$ are transmitted simultaneously by the antennas at instant $t$ and $t+T$ according to Table 1 ; where $T$ is the duration of a symbol as discussed in [23].

The gain of two wireless links or multipath fading factors is complex expressed as: $h_{1}=\alpha_{1} \mathrm{e}^{j \theta_{1}}$ and $h_{2}=\alpha_{2} \mathrm{e}^{j \theta_{2}}$. The entire scenario of Alamouti scheme is visualized from Figure 1.

The complex received signal at time $t^{\prime}>t$ is expressed as

$$
\tilde{x}_{1}=\alpha_{1} \mathrm{e}^{j \theta_{1}} \tilde{S}_{1}+\alpha_{2} \mathrm{e}^{j \theta_{2}} \tilde{S}_{2}+W_{1}
$$

The corresponding received signal at time $t^{\prime}+T$ is

$$
\tilde{x}_{2}=-\alpha_{1} \mathrm{e}^{j \theta_{1}} \tilde{S}_{2}^{*}+\alpha_{2} \mathrm{e}^{j \theta_{2}} \tilde{S}_{1}^{*}+W_{2}
$$

where $W_{1}$ and $W_{2}$ are the AWGN noise.

From (1) and (2),

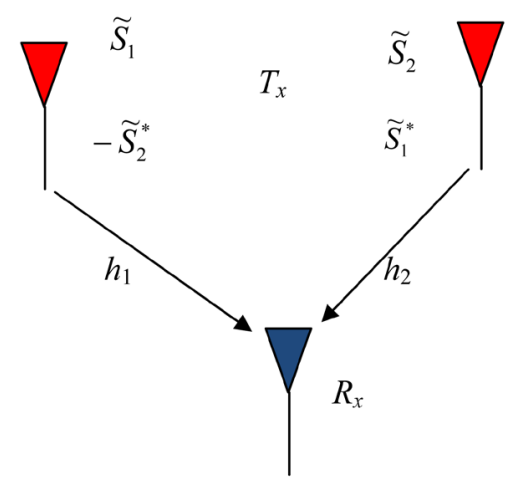

Figure 1. Alamuoti scheme in simplest form.

Table 1. Symbol sequence of two antenna elements.

\begin{tabular}{ccc}
\hline Time & $T_{x 1}$ & $T_{x 2}$ \\
\hline$t$ & $\tilde{S}_{1}$ & $\tilde{S}_{2}$ \\
$t+T$ & $-\tilde{S}_{2}^{*}$ & $\tilde{S}_{1}^{*}$ \\
\hline
\end{tabular}




$$
\left[\begin{array}{c}
\tilde{x}_{1} \\
\tilde{x}_{2}^{*}
\end{array}\right]=\left[\begin{array}{cc}
\alpha_{1} \mathrm{e}^{j \theta_{1}} & \alpha_{2} \mathrm{e}^{j \theta_{2}} \\
\alpha_{2} \mathrm{e}^{-j \theta_{2}} & -\alpha_{1} \mathrm{e}^{-j \theta_{1}}
\end{array}\right]\left[\begin{array}{c}
\tilde{S}_{1} \\
\tilde{S}_{2}
\end{array}\right]+\left[\begin{array}{c}
\tilde{W}_{1} \\
\tilde{W}_{2}^{*}
\end{array}\right]
$$

Now, the output of the linear combiner using the concept of channel knowledge of [24] [25],

$$
\begin{gathered}
{\left[\begin{array}{l}
\tilde{y}_{1} \\
\tilde{y}_{2}
\end{array}\right]=\left[\begin{array}{cc}
\alpha_{1} \mathrm{e}^{-j \theta_{1}} & \alpha_{2} \mathrm{e}^{j \theta_{2}} \\
\alpha_{2} \mathrm{e}^{-j \theta_{2}} & -\alpha_{1} \mathrm{e}^{j \theta_{1}}
\end{array}\right]\left[\begin{array}{c}
\tilde{x}_{1} \\
\tilde{x}_{2}^{*}
\end{array}\right]} \\
\Rightarrow\left[\begin{array}{c}
\tilde{y}_{1} \\
\tilde{y}_{2}
\end{array}\right]=\left[\begin{array}{cc}
\alpha_{1} \mathrm{e}^{-j \theta_{1}} & \alpha_{2} \mathrm{e}^{j \theta_{2}} \\
\alpha_{2} \mathrm{e}^{-j \theta_{2}} & -\alpha_{1} \mathrm{e}^{j \theta_{1}}
\end{array}\right]\left(\left[\begin{array}{cc}
\alpha_{1} \mathrm{e}^{j \theta_{1}} & \alpha_{2} \mathrm{e}^{j \theta_{2}} \\
\alpha_{2} \mathrm{e}^{-j \theta_{2}} & -\alpha_{1} \mathrm{e}^{-j \theta_{1}}
\end{array}\right]\left[\begin{array}{c}
\tilde{S}_{1} \\
\tilde{S}_{2}
\end{array}\right]+\left[\begin{array}{c}
\tilde{W}_{1} \\
\tilde{W}_{2}^{*}
\end{array}\right]\right) \\
\Rightarrow\left[\begin{array}{c}
\tilde{y}_{1} \\
\tilde{y}_{2}
\end{array}\right]=\left(\begin{array}{ll}
\left.\alpha_{1}^{2}+\alpha_{2}^{2}\right) \\
1 & 0 \\
0 & 1
\end{array}\right]\left[\begin{array}{c}
\tilde{S}_{1} \\
\tilde{S}_{2}
\end{array}\right]+\left[\begin{array}{cc}
\alpha_{1} \mathrm{e}^{j \theta_{1}} & \alpha_{2} \mathrm{e}^{j \theta_{2}} \\
\alpha_{2} \mathrm{e}^{-j \theta_{2}} & -\alpha_{1} \mathrm{e}^{-j \theta_{1}}
\end{array}\right]\left[\begin{array}{c}
\tilde{W}_{1} \\
\tilde{W}_{2}^{*}
\end{array}\right] \\
\therefore\left[\begin{array}{c}
\tilde{y}_{1} \\
\tilde{y}_{2}
\end{array}\right]=\left(\alpha_{1}^{2}+\alpha_{2}^{2}\right)\left[\begin{array}{c}
\tilde{S}_{1} \\
\tilde{S}_{2}
\end{array}\right]+\left[\begin{array}{c}
n_{1} \\
n_{2}
\end{array}\right]
\end{gathered}
$$

In $\tilde{y}_{1}$, there is no component of $\tilde{S}_{2}$ and in $\tilde{y}_{2}$, there is no component of $\tilde{S}_{1}$, hence the system is fully orthogonal. The simulation of entire Alamouti scheme under fading channel can be represented by the flowchart of Figure 2.

\subsection{Algorithm}

This subsection provides the algorithm of image processing and transmission of the image through fading channel.

1. Select an RGB image and convert it to grayscale. Resize the image as the size of $N \times N$.

2. Covert the image to a column vector $\Gamma$ of size of $1 \times N^{2}$.

3. Divide the amplitude of each pixel of column vector by 16 and store the remainder $R(i)$ and quotient $Q(i)$ of $i$ th pixel where $i=1,2,3, \cdots, N^{2}$ using the concept of [26].

4. Since the amplitude of each pixel is in the range of $0-255$ therefore both $R(i)$ and $Q(i)$ has the value in the range of $0-15$ hence we can use the values as the constellation points of 16-QAM.

5. Assign each value of $R(i)$ and $Q(i)$ against a constellation point of 16-QAM.

6. Transmit the constellation points or symbols against corresponding values of $R(i)$ and $Q(i)$ thorough a Alamouti simulator with Rayleigh fading channel contaminated by AWGN. The flowchart of simulator is shown in Figure 2.

7. Recover each constellation points using orthogonal space diversity algorithm at the receiving end.

8. Convert the constellation points to $Q(i)$ and $R(i)$.

9. Evaluate the amplitude of pixel using $\Gamma(i)=16 * Q(i)+R(i)$.

10. Convert the column vector $\Gamma$ to the matrix $N \times N$ in the reverse way of step 2 .

11. Smooth the image using DWT with a threshold value.

We can apply RSA algorithm of [27] [28] on $R(i)$ and $Q(i)$ at step 5 to achieve secured communication.

\section{Results}

Let us first select a test image (main gate of Jahangirnagar University campus) and apply the algorithm on it for the fading channel of average SNR of 12, 15, 20, 25 and $30 \mathrm{~dB}$. The corresponding results are shown in Figures 3-7. The recovered image is found appreciable at a glance for $20 \mathrm{~dB}$ or above. The quality of received image improves at higher SNR is visualized from the figures. We can get better results by applying lower modulation scheme like, BPSK, QPSK, 8-PSK instead of 16-QAM, although throughput is higher at higher modulation scheme. We measure the BER of the same image (main gate of JU campus) for four different modulation schemes shown in Figure 8, where we get the conventional results, i.e. performance is better for lower modulation scheme. Actually the algorithm has very small effect on different images in context of BER. The phenomenon is shown in Table 2, where we select 6 different images and measure BER under 16-QAM taking SNR 


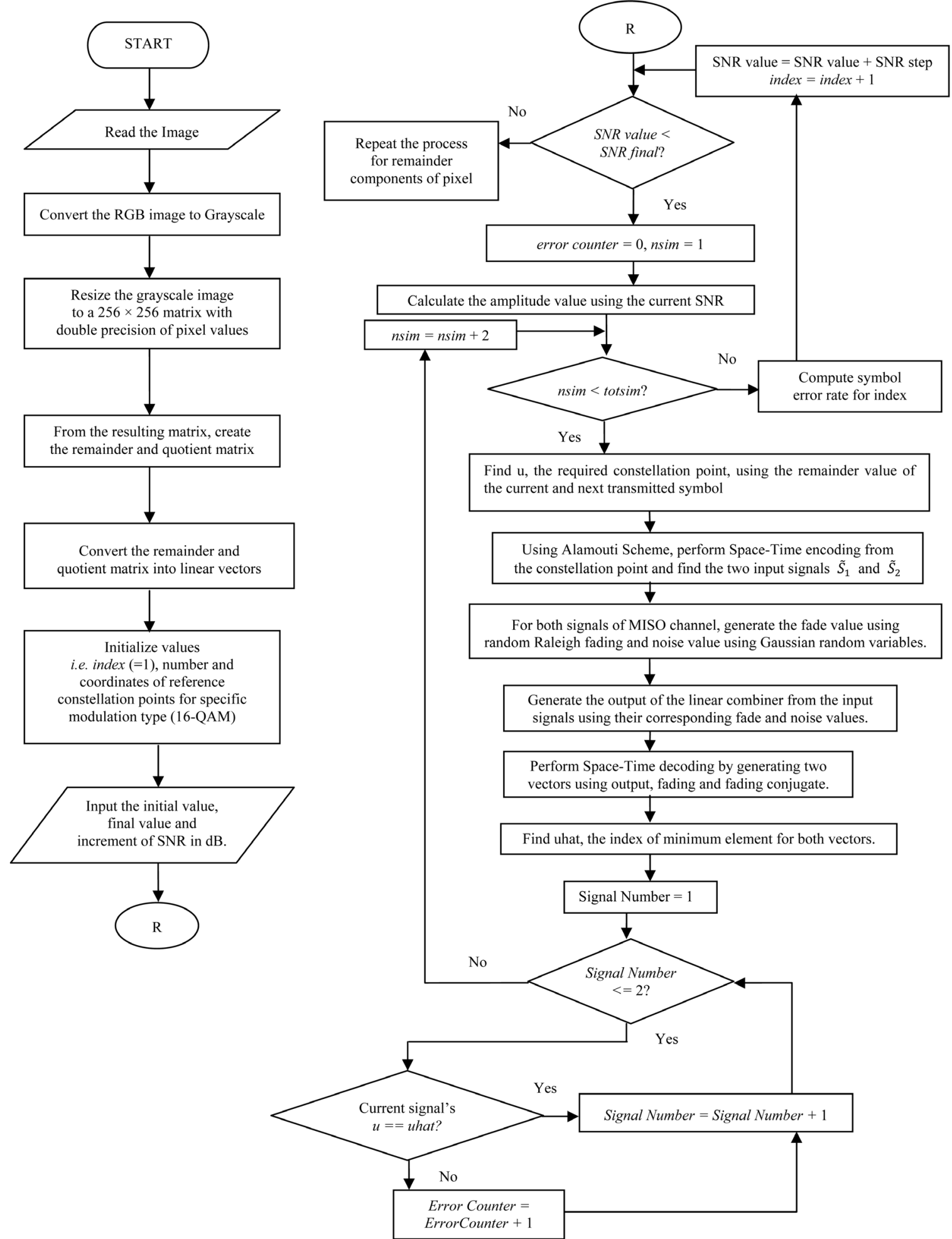

\section{Figure 2. Flowchart of Alamouti Simulator.}

from 12 to $30 \mathrm{~dB}$. Finally, for secured communication we apply RSA algorithm on $Q(i)$ and $R(i)$ of four images. The corresponding results are shown in Figure 9, where we show the original RGB image, its grayscale version, encrypted image and recovered/decrypted image. 

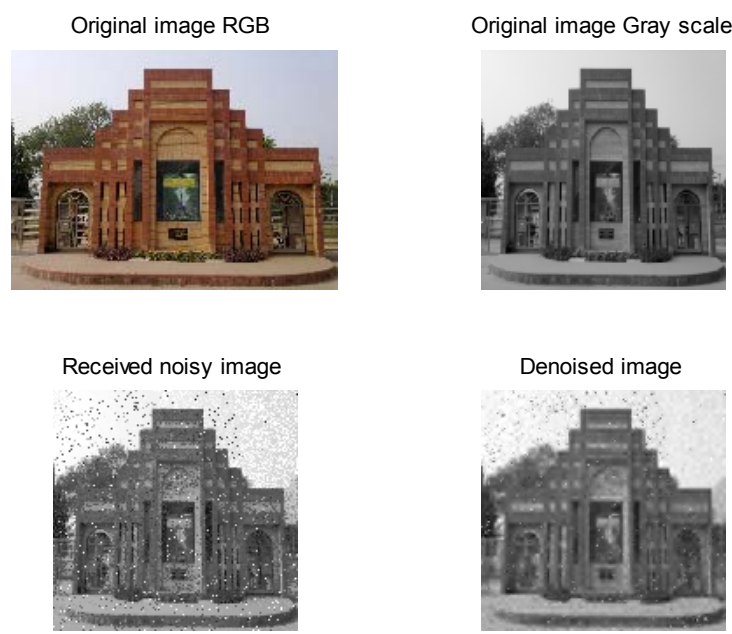

Figure 3. Image recovery at SNR of $12 \mathrm{~dB}$ using 16-QAM.
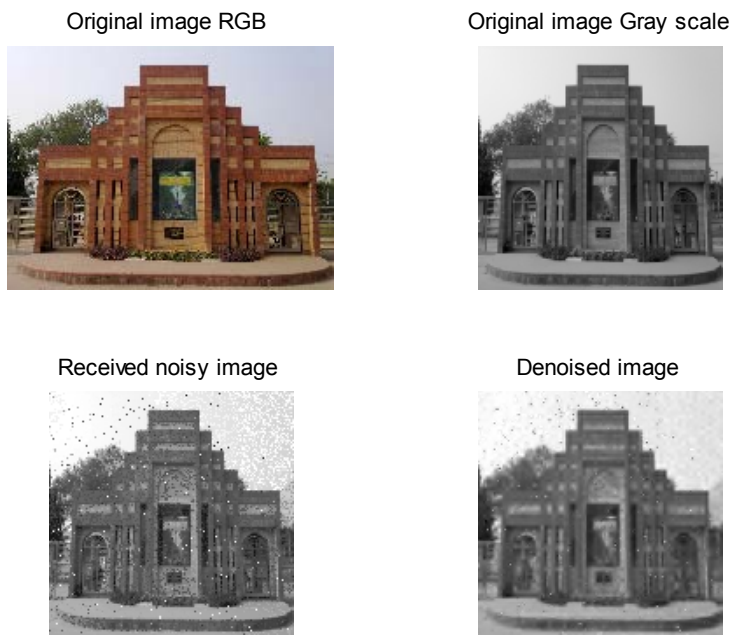

Denoised image

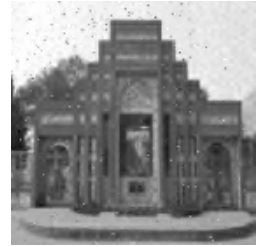

Figure 4. Image recovery at SNR of $15 \mathrm{~dB}$ using 16-QAM.
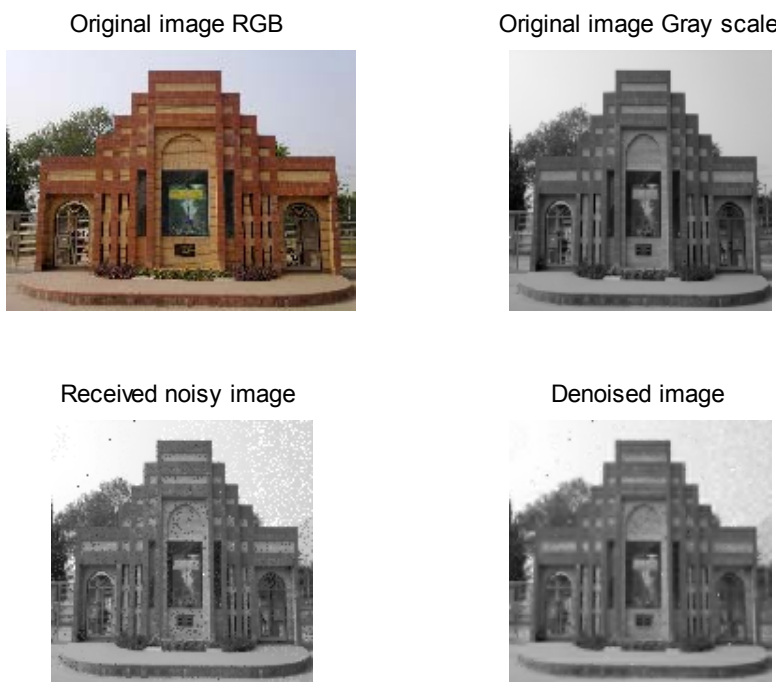

Figure 5. Image recovery at SNR of 20dB using 16-QAM. 


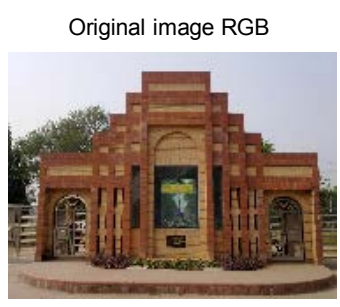

Original image Gray scale

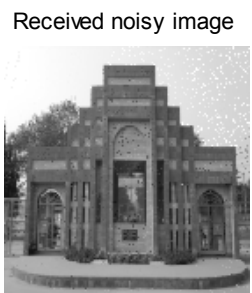

Denoised image

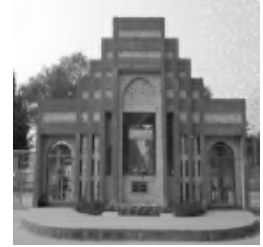

Figure 6. Image recovery at SNR of $25 \mathrm{~dB}$ using 16-QAM.
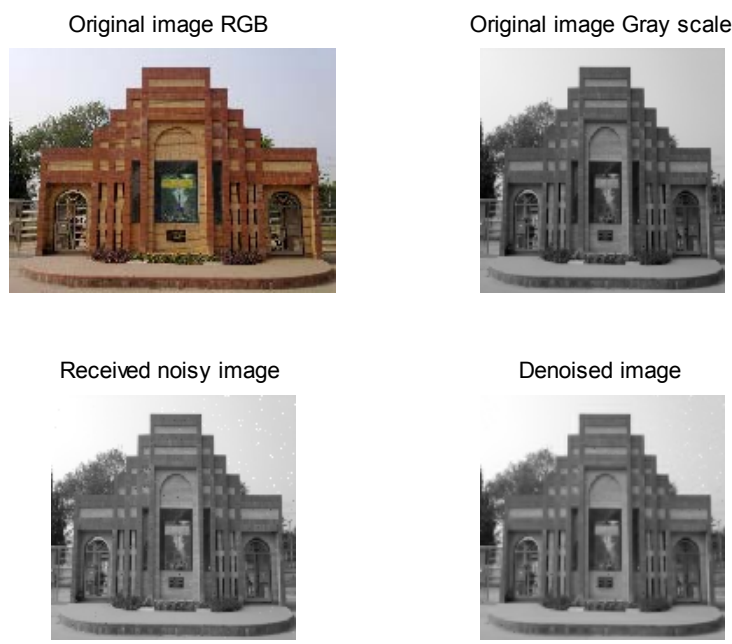

Figure 7. Image recovery at SNR of 30dB using 16-QAM.

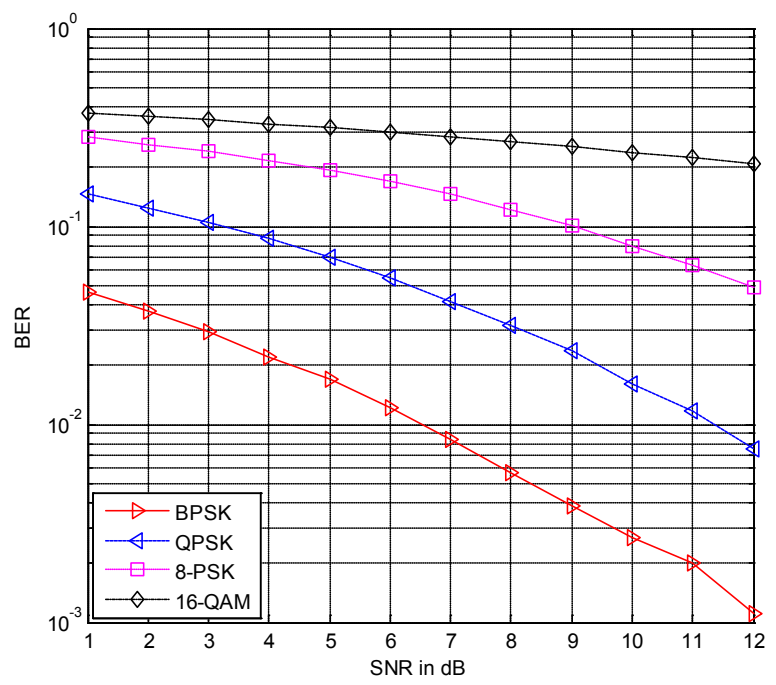

Figure 8. BER of the image of JU Gate under four different modulation schemes. 

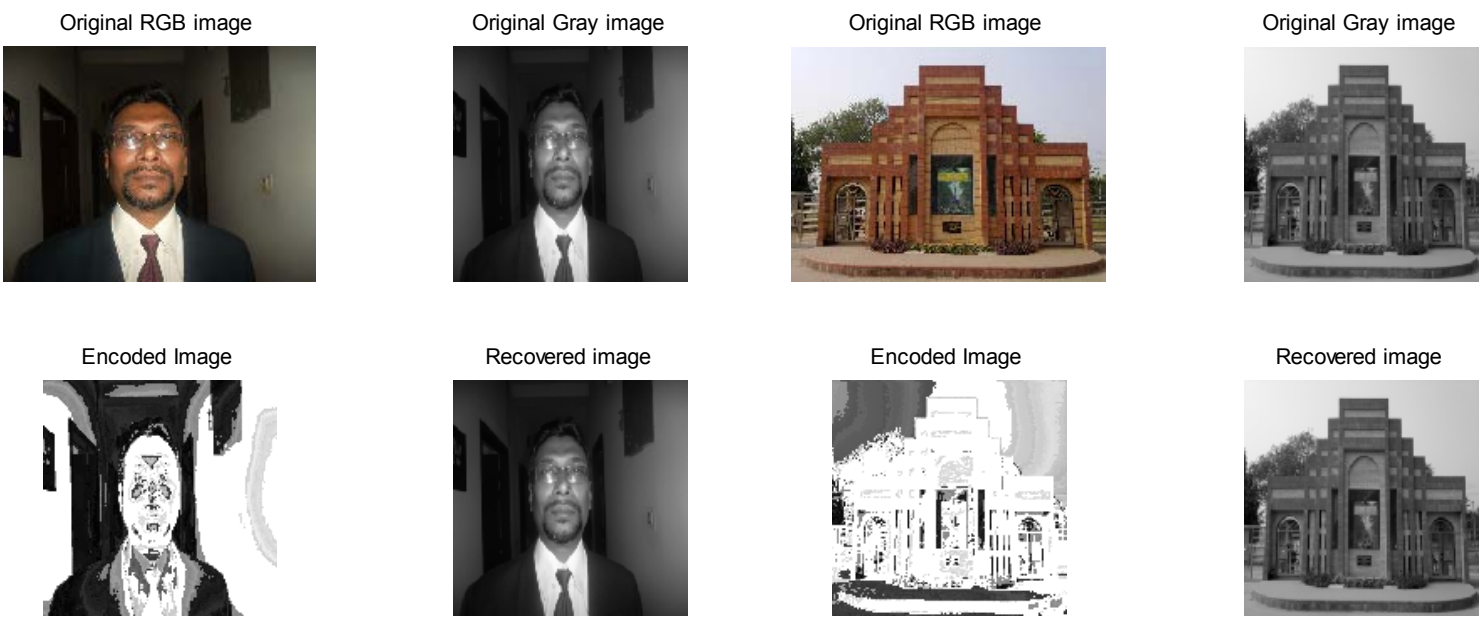

(a)

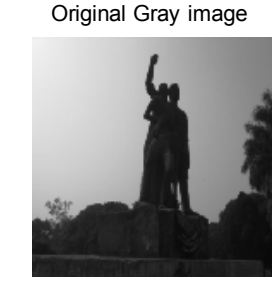

Original RGB image
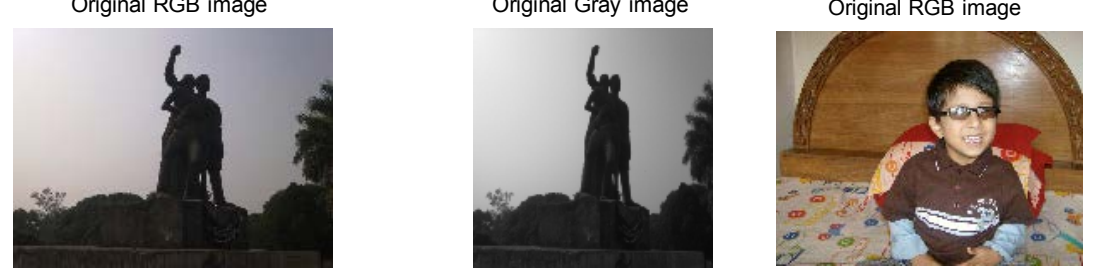

(b)
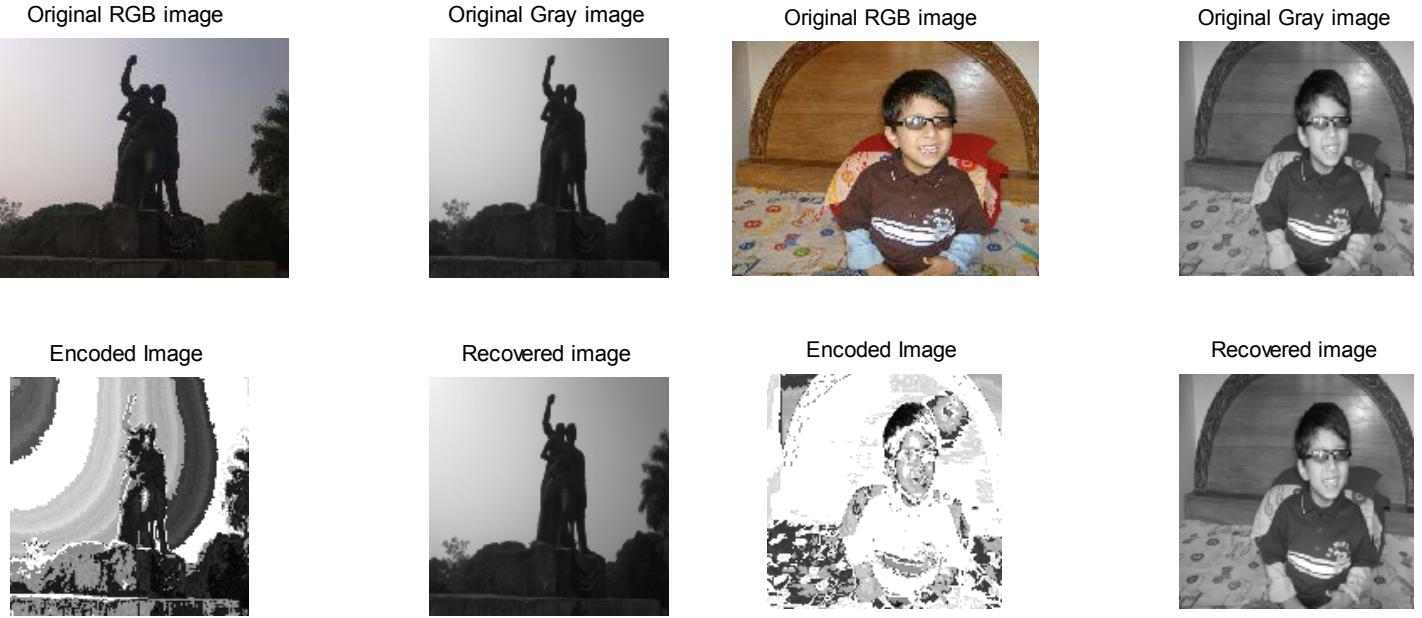

(c)

(d)

Figure 9. Encryption and decryption of image.

Table 2. BER of six test images under 16-QAM.

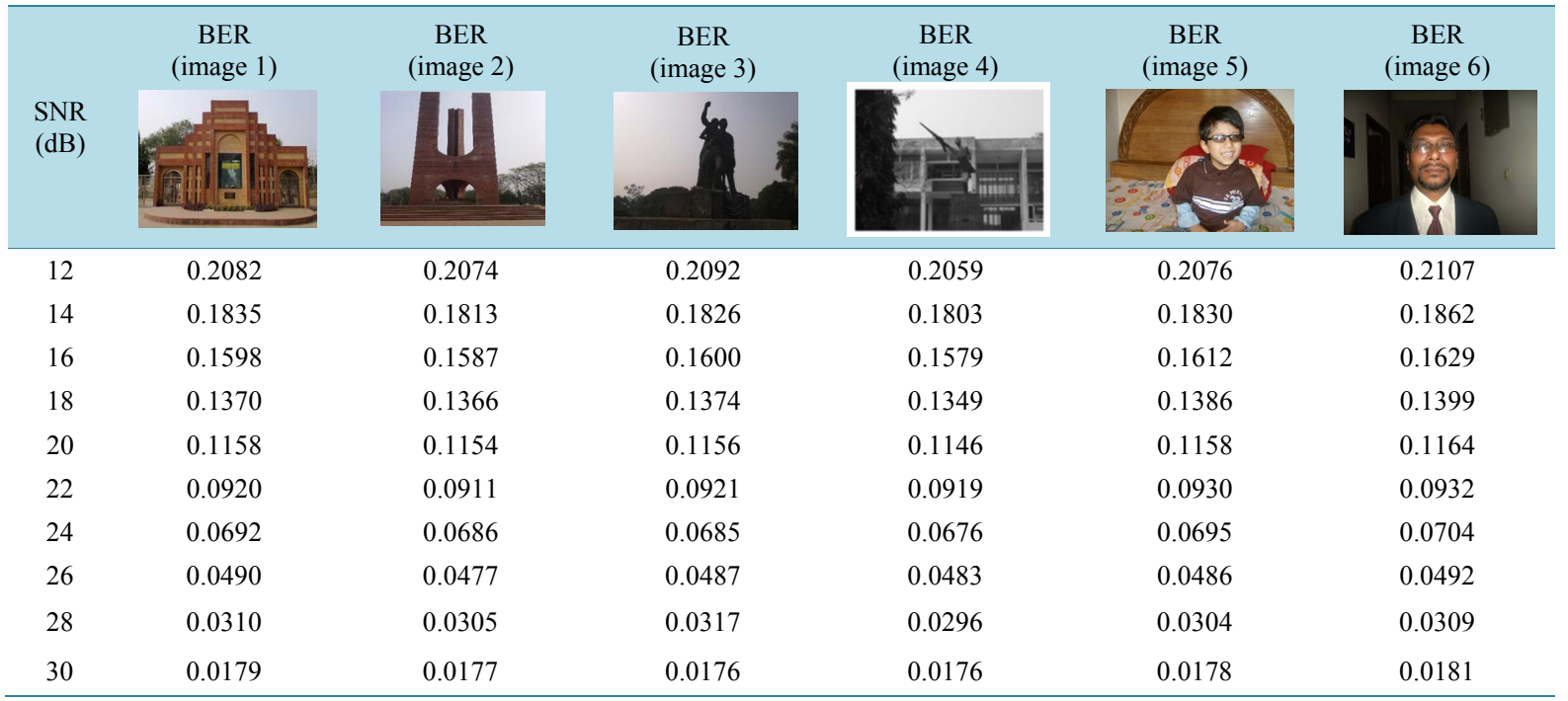




\section{Conclusion}

In this paper, we are able to recover an image transmitting through Alamouti channel with incorporation of RSA algorithm; where the quality of the image depends on the SNR of the channel. Although we reveal the results for grayscale images, we can also do the similar job for RGB images as well, sending the R, G and B components separately, then combining and at receiving end. We can apply channel coding scheme like linear block code, convolutional code or CRC (Cyclic Redundancy Code) scheme to enhance the performance of the system. MIMO and combining scheme (maximal ratio combining, equal gain, selection combining) can be considered an alternate technique of improvement of the system.

\section{References}

[1] Zhang, J.K., Chen, S., Mu, X.M. and Hanzo, L. (2011) Joint Channel Estimation and Multiuser Detection for SDMA/OFD Based on Dual Repeated Weighted Boosting Search. IEEE Transactions on Vehicular Technology, 60, 3265-3275. http://dx.doi.org/10.1109/TVT.2011.2161356

[2] Zhu, H.L., Xia, B. and Tan, Z.H. (2011) Performance Analysis of Alamouti Transmit Diversity with QAM in Imperfect Channel Estimation. IEEE Journal on Selected Areas in Communications, 29, 1242-1248.

[3] Alamouti, S. (1998) A Simple Transmit Diversity Technique for Wireless Communications. IEEE Journal on Selected Areas in Communications, 16, 1451-1458. http://dx.doi.org/10.1109/49.730453

[4] Wang, X.Y. and Wang, J. (2004) Effect of Imperfect Channel Estimation on Transmit Diversity in CDMA Systems. IEEE Transactions on Vehicular Technology, 53, 1400-1412.

[5] Derryberry, R.T., Gray, S.D., Ionescu, D.M., Mandyam, G. and Raghothaman, B. (2002) Transmit Diversity in 3G CDMA Systems. IEEE Communications Magazine, 40, 68-75. http://dx.doi.org/10.1109/35.995853

[6] Alam, M.M., Islam, A.Z.M.T. and Ullah, S.E. (2011) Performance Analysis of a Concatenated LDPC Coded Alamouti Based STBC-OFDM System on Text Message Transmission. International Journal of Computer Science, Engineering and Applications (IJCSEA), 1, 1-9.

[7] Ahmed, T., Ali, M.M. and Ullah, S.E. (2012) BER Performance Analysis of a STBC Encoded Secured Multiuser MIMO-OFDM Wireless Communication System. International Journal of Hybrid Information Technology, 5, 19-30.

[8] Anoh, K.O.O., Ochonogor, O., Abd-Alhameed, R.A.A., Jones, S.M.R. and Mapuka, T.T. (2014) Improved Alamouti STBC Multi-Antenna System Using Hadamard Matrices. International Journal of Communications, Network and System Sciences, 7, 83-89. http://dx.doi.org/10.4236/ijens.2014.73010

[9] Sklar, B. (2001) Digital Communications: Fundamentals and Applications. 2nd Edition, Prentice Hall, Upper Saddle River.

[10] Ryan, W.E. and Lin, S. (2009) Channel Codes: Classical and Modern. Cambridge University Press, Cambridge, UK, 4.

[11] Xia, B. and Wang, J. (2005) Effect of Channel Estimation Error on QAM Systems with Antenna Diversity. IEEE Transactions on Communications, 53, 481-488.

[12] Gu, D. and Leung, C. (2003) Performance Analysis of Transmit Diversity Scheme with Imperfect Channel Estimation. IEEE Electronics Letters, 39, 402-403. http://dx.doi.org/10.1049/el:20030243

[13] Stallings, W. (2005) Cryptography and Network Security Principles and Practices. 4th Edition, Prentice-Hall, New Jersey.

[14] Saeed, A., Xu, H.J. and Quazi, T. (2014) Alamouti Space-Time Block Coded Hierarchical Modulation with Signal Space Diversity and MRC Reception in Nakagami-m Fading Channel. IET Communications, 8, 516-524. http://dx.doi.org/10.1049/iet-com.2013.0519

[15] Fang, L., Bi, G. and Kot, A.C. (2000) New Method of Performance Analysis for Diversity Reception with Correlated Rayleigh-Fading Signals. IEEE Transactions on Vehicular Technology, 49, 1807-1812. http://dx.doi.org/10.1109/25.892585

[16] Ahmed, T., Anower, M.S., Sarkar, M.Z.I. and Ali, M.M. (2013) Investigation of Correlated Rayleigh Fading Channel with Alamouti's STBC-MRC System. International Journal of Advanced Science and Technology, 58, 65-74. http://dx.doi.org/10.14257/ijast.2013.58.06

[17] Perişoară, L.A. (2012) BER Analysis of STBC Codes for MIMO Rayleigh Flat Fading Channels. Telfor Journal, 4, 78-82.

[18] Bhatnagar, M.R., Vishwanath, R. and Bhatnagar, V. (2007) Performance Analysis of Space-Time Block Codes in Flat Fading MIMO Channels with Offsets. EURASIP Journal on Wireless Communications and Networking, 2007, Article ID: 030548 . 
[19] Badic, B., Herdin, M., Weinrichter, H. and Rupp, M. (2004) Quasi-Orthogonal Space-Time Block Codes on Measured MIMO Channels. Joint IST Workshop on Mobile Future, 2004 and the Symposium on Trends in Communications, Bratislava, 24-26 October 2004, 17-20.

[20] Badic, B., Rupp, M. and Weinrichter, H. (2004) Quasi-Orthogonal Space-Time Block Codes for Data Transmission over Four and Eight Transmit Antennas with Very Low Feedback Rate. 5th International ITG Conference on Source and Channel Coding (SCC), Erlangen, 14-16 January 2004, 157-164.

[21] Park, U., Kim, Y. and Kim, S. (2010) A New Result on Turbo Coded QO-STBC Schemes. IEEE Communications Letters, 14, 199-201. http://dx.doi.org/10.1109/LCOMM.2010.03.092404

[22] Chaudhary, S. and Patil, A.J. (2012) Performance Analysis of Mimo-Space Time Block Coding with Different Modulation Techniques. ICTACT Journal on Communication Technology, 3, 510-514.

[23] El-Astal, M.T.O., Abu-Hudrouss, A.M., Salmon, B.P. and Olivier, J.C. (2015) An Adaptive Transmission Protocol for Exploiting Diversity and Multiplexing Gains in Wireless Relaying Networks. EURASIP Journal on Wireless Communications and Networking, 2015, 1-15.

[24] Gupta, S., Dutta, R.K. and Tiwari, A.C. (2014) Performance Analysis of Alamouti and Orthogonal Space-Time Block Codes in MIMO System under Rayleigh Fading Scenario. International Journal of Advanced Research in Electrical, Electronics and Instrumentation Engineering, 3, 12423-12429.

[25] Rao, V. and Malavika, T. (2014) Performance Analysis of MIMO-OFDM for Multiple Antennas. International Journal of Advanced Research in Electrical, Electronics and Instrumentation Engineering, 3, 9349-9355.

[26] Jassim, F.A. and Qassim, H.E. (2012) Five Modulus Method for Image Compression. Signal \& Image Processing: An International Journal (SIPIJ), 3, 19-28.

[27] Ayele, A.A. and Sreenivasarao, V. (2013) Modified RSA Encryption Technique Based on Multiple Public Keys. International Journal of Innovative Research in Computer and Communication Engineering, 1, 859-864.

[28] Jamgekar, R.S. and Joshi, G.S. (2013) File Encryption and Decryption Using Secure RSA. International Journal of Emerging Science and Engineering (IJESE), 1, 11-14. 